\title{
PRODUCTIVIDAD Y CALIDAD DE FIBRA DE VARIEDADES COONVENCIONALES DE ALGODÓN EN LA COMARCA LAGUNERA, MÉXICO
}

\author{
PRODUCTIVITY AND FIBER QUALITY OF CONVENTIONAL COTTON \\ VARIETIES AT THE COMARCA LAGUNERA, MEXICO
}

\author{
Olga Bonilla-Barrientos' ${ }^{1}$ Enrique Hernández-Leal ${ }^{2 *}$, José Verastegui-Chávez², \\ Jorge Maltos-Buendía ${ }^{2}$, Edgardo Bautista-Ramírez ${ }^{3}$, \\ Aurelio Hernández-Bautista' y Luis M. Isidro-Requejo²
}

\begin{abstract}
${ }^{1}$ Colegio de Postgraduados, Campus Montecillo, Montecillo, Texcoco, Estado de México, México. ${ }^{2}$ Instituto Nacional de Investigaciones Forestales, Agrícolas y Pecuarias (INIFAP), Campo Experimental La Laguna, Matamoros, Coahuila, México. ${ }^{3}$ INIFAP, Campo Experimental Centro Altos de Jalisco, Tepatitlán de Morelos, Jalisco, México.
\end{abstract}

*Autor de correspondencia (hernandez.enrique@inifap.gob.mx; ki29_ke1185@hotmail.com)

\section{RESUMEN}

Por su alta calidad de fibra, precocidad y menor tamaño vegetativo el algodón mexicano (Gossypium hirsutum L.) es el más cultivado en el mundo, aporta casi $90 \%$ de la producción mundial. México se ubica en el decimotercer lugar entre los principales países productores de algodón, del cual India, China, Estados Unidos de América, Brasil y Pakistán concentran el $77 \%$ de la producción mundial. En México, desde hace más de 20 años se siembra algodón transgénico por su tolerancia al ataque del complejo de larvas del algodonero y por su resistencia a herbicidas como glifosato, glufosinato de amonio y dicamba; sin embargo, algunos agricultores consideran que el algodón transgénico no es rentable y estarían dispuestos a volver a sembrar variedades convencionales si éstas estuvieran disponibles en el mercado. Por lo anterior, en este estudio se evaluaron las variedades convencionales de algodón Cian Precoz, Cian 95, Juárez 91, Laguna 89 y Nazas, junto con el testigo comercial Delta Pine en relación con su comportamiento productivo. El sistema de producción fue en surcos con distanciamiento de $0.76 \mathrm{~m}$ y nueve plantas por $\mathrm{m}$ lineal, para contar con una población de 115,000 plantas $\mathrm{ha}^{-1}$. Se midió el rendimiento de algodón en hueso y fibra, la precocidad a la primera cosecha, los componentes del rendimiento y la calidad de fibra. Se detectaron diferencias significativas $(P \leq 0.05)$ entre variedades en todas las variables debido a amplia variación genética existente. Delta Pine produjo el mayor rendimiento (3906 $\mathrm{kg} \mathrm{ha}^{-1}$ ) y fue superior al resto de las variedades en peso de fibra, semilla y peso de fibra y semilla. En relación con los requerimientos de calidad de fibra para la industria textil, todos los materiales evaluados cumplen con los requisitos mínimos de longitud, resistencia al rompimiento e índice de micronaire.

Palabras clave: Gossypium hirsutum L., componentes del rendimiento, productividad de algodón, propiedades de la fibra, variación genética.

\section{SUMMARY}

Due to its high fiber quality, earliness and smaller vegetative size, Mexican cotton (Gossypium hirsutum L.) is the most cultivated worldwide, providing almost $90 \%$ of world production. Mexico ranked thirteenth among the main cotton-producing countries, of which India, China, the United States of America, Brazil and Pakistan account for $77 \%$ of world production. In Mexico, transgenic cotton has been planted for more than 20 years because of its tolerance to the attack of the cotton larvae complex and its resistance to herbicides such as glyphosate, ammonium glufosinate and dicamba; however, some farmers consider that transgenic cotton is not profitable and would be willing to plant conventional varieties if they were available in the market. Therefore, in this study, the conventional Cian Precoz, Cian 95, Juárez 91, Laguna 89 and Nazas cotton varieties were evaluated, along with the Delta Pine commercial control, in relation to their productive performance. The production system was in rows $0.76-\mathrm{m}$ apart, and nine plants per linear $\mathrm{m}$, to have a population of 115,000 plants ha-1. Traits such as seed and lint yield, earliness to first harvest, yield components and fiber quality were measured. Significant differences $(P \leq 0.05)$ were detected between varieties for all the traits due to the wide genetic variation. Delta Pine produced the highest yield (3906.1 $\left.\mathrm{kg} \mathrm{ha}^{-1}\right)$; it was also superior to the rest of varieties in lint, seed and lint plus seed weight. Regarding the fiber quality requirements for the textile industry, all the materials evaluated meet the minimum requirements in length, breaking strength and micronaire index.

Index words: Gossypium hirsutum L., cotton productivity, fiber properties, genetic variation, yield components.

\section{INTRODUCCIÓN}

Por la fibra que produce (Poelham y Allen, 2003), el algodón (G. hirsutum L.) es considerado el producto agrícola no alimentario de mayor importancia a nivel mundial (SAGARPA y FAO, 2014) y representa alrededor del $30 \%$ de las fibras consumidas en la industria textil mundial (FAO, 2018); es cultivado en zonas tropicales y subtropicales del mundo (Vinent y Fajardo, 2007) en más de 50 países. G. hirsutum y $G$. barbadense son las especies que aportan más del $99 \%$ del abastecimiento mundial (Ashraf, 2002), y de estas dos, Gossypium hirsutum, o algodón mexicano, es el que más se cultiva en todo el mundo por su alta calidad de fibra en la fabricación de telas (Pérez et al., 2011; Poelham y Allen, 2003), con un aporte de casi 90 \% de la producción (SIAP, 2016).

Los principales países productores de algodón son India, China, Estados Unidos de América, Brasil y Pakistán; los cuales concentran $77 \%$ de la producción mundial (USDA, 2017). México se ubicó en el decimotercer lugar 
con un volumen de 487,914 t, lo que representó el 0.7\% de la producción mundial (SAGARPA, 2016), donde los principales estados productores son Chihuahua, Baja California, Coahuila, Tamaulipas, Sonora y Durango.

Alrededor del $96 \%$ de la semilla de algodón que se siembra en el mundo es transgénica y cuenta con la tecnología Bt (Bolgard II) que expresa las toxinas Cry1Ac y Cry2Ab (Nava-Camberos et al., 2018). En México, los algodones transgénicos se utilizan desde hace más de 20 años (Martínez-Carrillo y Díaz-López, 2005; Rocha-Munive et al., 2018; Traxler et al., 2003) para resistir los ataques del gusano rosado (Pectinophora gossypiella Sunders) y tabacalero (Heliothis virescens F.) principalmente, además del gusano bellotero (Helicoverpa zea Boddie) y gusano soldado (Spodoptera exigua) (Nava-Camberos et al., 2018).

No obstante, la siembra de semilla transgénica puede ocasionar que las plagas generen resistencia debido a la siembra intensiva de algodón Bt (Wu y Guo, 2005; Wu et al., 2008), por lo que se han desarrollado medidas para retrasar dicha resistencia, como la plantación de zonas refugio no Bt junto con la siembra de algodón Bt (Gould et al., 2002; Mendelsohn et al., 2003), donde pueden ser aprovechadas las variedades convencionales.

En un estudio reciente, Rocha-Munive et al. (2018) evaluaron el impacto del algodón genéticamente modificado después de 20 años de ser cultivado en México y encontraron que, a pesar del costo de producción, 80 \% de los agricultores está muy satisfechos con el uso de las variedades GM, 11 \% está moderadamente satisfecho y $9 \%$ no está satisfecho; además, $10 \%$ de los agricultores consideraron que el algodón GM no es rentable y 40 $\%$ estarían dispuestos a volver a sembrar variedades convencionales si estuvieran disponibles.

En México, el Instituto Nacional de Investigaciones Forestales, Agrícolasy Pecuarias (INIFAP) fue el encargado de generar variedades convencionales de algodón desde 1960 hasta 1995 mediante el programa de mejoramiento genético, el cual liberó 20 variedades (Pérez et al., 2011). Las últimas variedades que se liberaron para el estado de Chihuahua fueron Cian Precoz 2 y 3 en 1995 (Espinosa et al., 2004). Actualmente, las únicas variedades que se distribuyen y comercializan para la siembra de algodón en México son transgénicas y provienen de la empresa transnacional Monsanto. Son producidas en Estados Unidos de América y la semilla se comercializa a un alto precio, lo que incrementa los costos de producción para pequeños y medianos productores; además, se requiere de maquinaria especializada para la siembra, lo que resulta en altos costos de producción (Rocha-Munive et al., 2018); al respecto, la FAO (2018) menciona que el algodonero es una de las especies agrícolas en el mundo que consume cerca del $25 \%$ de todos los insecticidas.

Por lo antes mencionado, existe gran demanda de nuevas variedades de algodón con la finalidad de disminuir los costos de producción del cultivo para los medianos y pequeños productores, de ahí el interés de generar y evaluar nuevos materiales convencionales para abastecer la demanda de los productores, meta en la que sin duda los estudios de diversidad genética realizados en algodón, como los de CONABIO (Mastretta-Yanes et al., 2019) serán de gran utilidad. En este tenor, el objetivo del presente estudio fue evaluar el comportamiento productivo y la calidad de fibra de cinco variedades convencionales de algodón desarrolladas en el programa de mejoramiento genético del INIFAP para su uso comercial.

\section{MATERIALES Y MÉTODOS}

\section{Sitio experimental y material genético}

El presente trabajo se llevó a cabo en el ciclo primaveraverano de 2018 en el Campo Experimental La Laguna (CELALA) del INIFAP en Matamoros, Coahuila, México, cuya localización geográfica es $25^{\circ} 32^{\prime} \mathrm{N}$ y $103^{\circ} 14^{\prime} \mathrm{O}$ a $1150 \mathrm{msnm}$. Se evaluaron las variedades convencionales de algodón Cian Precoz, Cian 95, Juárez 91, Laguna 89 y Nazas, las cuales fueron obtenidas del programa de mejoramiento genético del INIFAP por el Dr. Arturo Palomo Gil'; además, se incluyó la variedad Delta Pine® como testigo.

\section{Manejo agronómico}

La siembra se realizó el 18 de abril de 2018 a una distancia de $0.76 \mathrm{~m}$ entre surcos y nueve plantas por metro lineal, para contar con una población de 115,000 plantas ha $^{-1}$. La dosis de fertilización utilizada fue 120N-40P-00K, aplicada antes del riego de siembra; posteriormente, se aplicaron tres riegos a los 52, 72 y 92 días después de la siembra (dds). El control de maleza y cosecha se realizaron manualmente, la primera cosecha se realizó a los 120 y la segunda a los 136 dds.

\section{Diseño y unidad experimental}

Se utilizó un diseño experimental de bloques completos al azar con tres repeticiones. La parcela experimental consistió de seis surcos de $6 \mathrm{~m}$ de longitud y las plantas muestreadas se obtuvieron de los dos surcos centrales. 


\section{Variables evaluadas}

Se registró el rendimiento de algodón (REN), de fibra (REF) y de semilla (RES) en $\mathrm{kg} \mathrm{ha}^{-1}$, además del porcentaje que éste representa del total cosechado (PPH), altura final de ocho plantas por parcela (APL) en $\mathrm{cm}$. Para los componentes de rendimiento, se cuantificaron el peso del capullo (PCA) en g, el cual se obtuvo al dividir el peso de la muestra entre el número de capullos, el número de capullos por planta (NCPP) se obtuvo de dividir el rendimiento de la parcela entre el número de plantas cosechadas y posteriormente entre el peso del capullo, contenido de fibra (CFI) en \% como la proporción que representa al peso de la fibra con respecto al peso de la muestra y el índice de semilla se obtuvo del peso de 100 semillas (ISE) en $\mathrm{g}$.

Para la calidad de la fibra se tomó una muestra aleatoria de 20 capullos por parcela, se separó la fibra de la semilla y se pesó. El análisis se realizó en el laboratorio de calidad de fibra del CELALA-INIFAP, donde se determinó a cada muestra la longitud de fibra (LON) en $\mathrm{mm}$, la resistencia (RES) en megapascales (MPa) y la finura (FIN) en índice de micronaire.

\section{Análisis estadístico}

Se realizó análisis de varianza a cada variable utilizando el procedimiento GLM de SAS (SAS Institute, 2011). Cuando se detectaron diferencias estadísticas entre variedades $(P \leq 0.05)$ se aplicó la prueba de separación de medias de Tukey $(P \leq 0.05)$. Finalmente, se realizo un análisis de correlaciones para determinar la asociación existente entre las variables.

\section{RESULTADOS Y DISCUSIÓN}

Se detectaron diferencias significativas $(P \leq 0.05)$ entre las variedades en las 12 variables evaluadas (Cuadro 1), debido a la amplia variación genética entre las variedades. Los mayores coeficientes de variación se observaron en el peso de fibra y semilla, NCPP, peso de fibra y peso de capullo, lo cual indica que la variabilidad se debe a diferencias genéticas intrínsecas (Nuez et al., 2008), ya que fueron evaluadas en las mismas condiciones. El resto de las variables mostró coeficientes de variación menores al $20 \%$, el más bajo fue para el contenido de fibra (6.1\%). Al respecto, Pimentel (1985) estableció que, en experimentos agrícolas realizados en campo, normalmente, el CV es considerado bajo cuando es inferior a $10 \%$, medio de 10 a $20 \%$, alto de 20 a $30 \%$ y muy alto cuando es mayor de $30 \%$.

\section{Rendimiento y sus componentes}

Los resultados de la prueba de medias indican que el testigo Delta Pine obtuvo el mayor rendimiento (3906.1 kg

Cuadro 1. Cuadrados medios del análisis de varianza de 12 caracteres evaluados en seis variedades convencionales de algodón en la Comarca Lagunera, México, 2018.

\begin{tabular}{lrrrrrr}
\hline FV & \multicolumn{1}{c}{ Variedad } & \multicolumn{1}{c}{ Rep } & \multicolumn{1}{c}{ Error } & CV (\%) & \multicolumn{1}{c}{ Media } & \multicolumn{1}{c}{ DE } \\
\hline Rendimiento & $112722.7 * *$ & $19767.7 * *$ & 123476.2 & 18.8 & 1980.3 & 1197.3 \\
Rendimiento de fibra & $6294290.5 * *$ & $1125662.3 * *$ & 23056.6 & 21.3 & 713.7 & 511.9 \\
Rendimiento de hueso & $11769126.0 * *$ & $2676064.6 * *$ & 41805.4 & 19.7 & 1038.0 & 704.9 \\
Peso de capullo & $19.5 * *$ & $5.8 *$ & 1.5 & 20.3 & 5.9 & 1.6 \\
Contenido de fibra & $14.9 * *$ & 7.1 & 6.0 & 6.1 & 40.4 & 2.6 \\
Índice de semilla & $8.2 * *$ & 1.1 & 1.0 & 9.8 & 10.3 & 1.8 \\
NCPP & $5303.0 * *$ & $856.7 * *$ & 102.1 & 38.8 & 26.1 & 20.2 \\
Altura de planta & $404.3 * *$ & $574.8 * *$ & 45.7 & 10.0 & 67.3 & 8.8 \\
Peso de fibra y hueso & $115676.1 * *$ & $19900.3 * *$ & 2125.5 & 44.8 & 102.9 & 93.9 \\
Finura & $2.5 * *$ & $3.9 * *$ & 0.2 & 10.1 & 4.8 & 0.1 \\
Resistencia & $4383.5 *$ & 4329.3 & 1800.0 & 7.0 & 604.4 & 44.9 \\
Longitud de fibra & $36.4 * *$ & $37.0 * *$ & 5.2 & 7.1 & 32.1 & 2.8 \\
GL & 5 & 2 & 85 & 92 & & \\
\hline
\end{tabular}

FV: fuente de variación, Rep: repeticiones, CV: coeficiente de variación, DE: desviación estándar, *: P $\leq 0.05, * *$ : P $\leq 0.01$, NCPP. número de capullos por planta, GL: grados de libertad. 
$\left.\mathrm{ha}^{-1}\right)$; además, fue superior al resto de las variedades en los componentes de rendimiento, peso de fibra, semilla y peso de fibra y semilla; por el contrario, Juárez 91 y Laguna 89 tuvieron un rendimiento de algodón en semilla de apenas 739.9 y $630.9 \mathrm{~kg} \mathrm{ha}^{-1}$, respectivamente; de la misma manera, dichas variedades mostraron los valores más bajos para las variables rendimiento de fibra (192.5 y $155.72 \mathrm{~kg} \mathrm{ha}^{-1}$ ) y rendimiento de semilla (293.67 y 228.31 $\mathrm{kg} \mathrm{ha}^{-1}$ ) (Cuadro 2). Los bajos rendimientos obtenidos con las variedades Laguna 89 y Juárez 91 pueden ser debidos a que son variedades obsoletas, obtenidas hace más de 30 años para condiciones ambientales distintas a las actuales (Arias et al., 2010; SNICS, 2012).

Palomo et al. (2014) reportaron que la media nacional de algodón semilla fue de $5000 \mathrm{~kg} \mathrm{ha}^{-1}$, mientras que la media regional en La Comarca Lagunera, reportado por GaytánMascorro et al. (2004), se ubicó en $4200 \mathrm{~kg} \mathrm{ha}^{-1}$ de algodón semilla y de $1500 \mathrm{~kg} \mathrm{ha}^{-1}$ de algodón fibra. Con base en estos valores, solamente Delta Pine fue ligeramente superior en algodón fibra, ya que las otras variedades tuvieron rendimientos muy bajos que podrían deberse a las condiciones ambientales como lluvia y temperatura que prevalecen durante el ciclo del cultivo (Jost y Cothren, 2000; Palomo-Gil et al., 2004).

Para el resto de los componentes de rendimiento, las variedades convencionales fueron superiores al testigo comercial; Juárez 91 tuvo el mayor peso de capullo con
$7.66 \mathrm{~g}$, lo que superó al resto de las variedades, que se ubicaron en un intervalo de 5.08 a 6.35 g; además, dicha variedad también tuvo el mayor índice de semilla. El testigo comercial Delta Pine presentó el mayor número de capullos por planta, lo cual se refleja en los rendimientos; al respecto, Worley et al. (1976) y Espitia et al. (2008) mencionaron que el número de capullos por unidad de superficie, el número de semillas por cápsula y la fibra determinan el rendimiento en algodón. Para el contenido de fibra o Ginning Out-Turn (GOT), Delta Pine tuvo el mayor porcentaje de fibra (42.26), mientras que Cian Precoz presentó el menor porcentaje (39.24); el resto no presentó diferencias estadísticas con respecto a Delta Pine. Godoy et al. (1998; Com. Pers.) ${ }^{1}$ mencionaron que las variedades convencionales Cian Precoz y Laguna 89 fueron superiores a la variedad transgénica NuCOTN 35B con 36.9 y $37 \%$, respectivamente, por lo que estos materiales tienen características superiores que algunas variedades transgénicas (Escobedo-Salas et al., 2004).

\section{Calidad de fibra}

Delta Pine fue la variedad con el valor más alto de finura con 5.59 micronaires, lo que la ubica, de acuerdo con la clasificación por instrumentos de alto volumen o HVI (CNSPA, 2012), como muy gruesa; por el contrario, Cian

'Godoy A. S., A. Palomo G. y E. A. García C. (1998) Validación de la variedad de algodonero NuCOTN 35B en la Comarca Lagunera. CELALA INIFAP. Matamoros, Coahuila, México. 2 p.

Cuadro 2. Comparación de características productivas de seis variedades convencionales de algodón en la Comarca Lagunera, México, ciclo agrícola primavera-verano de 2018.

\begin{tabular}{|c|c|c|c|c|c|c|c|c|c|c|c|c|c|}
\hline \multirow{3}{*}{$\frac{\text { Variable }}{\text { REN (kg ha-1) }}$} & \multicolumn{12}{|c|}{ Variedades } & \multirow{3}{*}{$\begin{array}{l}\text { DSH } \\
(0.05)\end{array}$} \\
\hline & \multicolumn{2}{|l|}{ Nazas 87} & \multicolumn{2}{|c|}{ Juárez 91} & \multicolumn{2}{|c|}{ Laguna 89} & \multicolumn{2}{|l|}{ Cian 95} & \multicolumn{2}{|c|}{ Cian Precoz } & \multicolumn{2}{|c|}{ Delta Pine } & \\
\hline & 2169.3 & $\mathrm{~b}$ & 739.9 & C & 630.9 & c & 2058 & $b$ & 2336 & $b$ & 3906.1 & a & \\
\hline $\operatorname{REF}\left(\mathrm{kg} \mathrm{ha}^{-1}\right)$ & 779.21 & $b$ & 192.5 & c & 155.72 & c & 725.67 & $b$ & 845.72 & $b$ & 1565.72 & a & 127.66 \\
\hline $\operatorname{REH}\left(\mathrm{kg} \mathrm{ha}^{-1}\right)$ & 1153.55 & bc & 293.67 & $d$ & 228.31 & $d$ & 1102.75 & c & 1292.92 & $b$ & 2133.93 & a & 171.89 \\
\hline PCA (g) & 5.14 & $b$ & 7.66 & a & 6.35 & b & 5.29 & $b$ & 5.08 & $b$ & 5.2 & $b$ & 1.31 \\
\hline $\mathrm{PPH}(\mathrm{g})$ & 131.86 & $b$ & 30.02 & $c$ & 25.54 & C & 109.22 & $b$ & 150.93 & $b$ & 259.73 & a & 49.47 \\
\hline CFI & 40.43 & $a b$ & 40.03 & $a b$ & 40.53 & $a b$ & 39.69 & $a b$ & 39.24 & $b$ & 42.26 & a & 2.63 \\
\hline ISE & 9.38 & $c$ & 11.36 & a & 10.18 & $a b$ & 10.46 & $a b$ & 9.67 & bc & 9.63 & $b c$ & 1.07 \\
\hline NCPP & 33.31 & $b$ & 9.4 & c & 9.38 & C & 29.86 & $b$ & 35.5 & $b$ & 58.62 & a & 10.84 \\
\hline APL (cm) & 71.15 & a & 61.1 & c & 63.33 & bc & 73.88 & a & 68.92 & $a b$ & 71.08 & a & 7.25 \\
\hline FIN (micronaire) & 4.78 & $\mathrm{bc}$ & 4.55 & bc & 4.62 & bc & 4.51 & c & 5.06 & $b$ & 5.59 & $a$ & 0.5181 \\
\hline RES (MPa) & 567.77 & $b$ & 611.15 & $a b$ & 614.19 & a & 618.93 & a & 603.5 & $a b$ & 600.15 & $a b$ & 45.52 \\
\hline LON (mm) & 30.8 & $b c$ & 33.19 & $a b$ & 31.79 & bc & 34.48 & a & 30.03 & $\mathrm{c}$ & 31.49 & bc & 2.46 \\
\hline
\end{tabular}

Medias con letras iguales en las filas no son estadísticamente diferentes (Tukey, 0.05). DSH: diferencia significativa honesta, REN: rendimiento de algodón, REF: rendimiento de fibra, REH: rendimiento de hueso, PCA: peso de capullo, PPH: peso de fibra y hueso, CFI: contenido de fibra, ISE: índice de semilla, NCPP. número de capullos por planta, APL: altura de planta, FIN: finura; RES: resistencia de fibra, LON: Iongitud de fibra. 
95, Juárez 91 y Laguna 89 tuvieron los valores más bajos, ubicándose en la clasificación como de finura media. Dichos valores pudieron estar influenciados durante el periodo de crecimiento por las condiciones ambientales como calidad de suelo, temperatura, precipitación pluvial, manejo, etc. y la presencia extrema de plantas o cápsulas (Estrada et al., 2008).

Con respecto a la resistencia, Cian 95, Laguna 89 y Juárez 91 tuvieron los valores más altos con 618.93, 614.19 y 611.15 MPa, respectivamente, mientras que Nazas 87 fue la de menor resistencia con 567.77 MPa. Para la longitud, Cian 95 fue la de mayor valor con $34.48 \mathrm{~mm}$, seguida de Juárez 91; Cian Precoz presentó el valor más bajo con 30.03 $\mathrm{mm}$. A pesar de que hubo diferencias estadísticamente significativas entre los materiales evaluados, la industria textil requiere como mínimo calidad de fibra de 26.7 a 27.2 $\mathrm{mm}$ de longitud, $517.5 \mathrm{MPa}$ de resistencia al rompimiento y entre 3.5 a 4.9 unidades en índices de micronaire o grosor de fibra (Estrada et al., 2008; Palomo et al., 2003), por lo que todos los materiales evaluados, excepto Delta Pine cumplen con dichos requisitos. Palomo et al. (2003) señalaron que variedades con fibra de alta calidad no tienen un sobreprecio en el mercado por la venta de su fibra, por lo que el productor no recibe ningún beneficio adicional; además, la longitud, resistencia y finura de la fibra se deben más a factores genéticos que a factores ambientales (Longenecker y Erie, 1968; Palomo-Gil et al., 2004).

En el Cuadro 3 se presenta la matriz de correlaciones de Pearson entre pares de variables. Se observa que 37 coeficientes fueron significativos; de ellos, 24 fueron positivos y 13 negativos; sin embargo, sólo 11 correlaciones fueron superiores a 0.60 .

El rendimiento de algodón en semilla estuvo altamente correlacionado con rendimiento de fibra $(r=0.99)$, rendimiento de semilla $(r=0.99)$ y peso de fibra y semilla $(r=0.91)$. Por su parte, rendimiento de fibra mostró una fuerte correlación con rendimiento de semilla $(r=0.99)$, número de capullos por planta $(r=0.90)$ y peso de fibra y semilla $(r=0.91)$. El rendimiento de semilla tuvo una alta correlación con número de capullos por planta $(r=0.91)$ y $\mathrm{PPH}(r=0.91)$. El número de capullos por planta exhibió una fuerte asociación con peso de fibra y semilla $(r=0.98)$. Finalmente, la variable finura, presentó una moderada correlación negativa con longitud de fibra $(r=-0.65)$. Todos estos valores concuerdan con los reportados por Scholl y Miller (1976), Dedaniya y Pethani (1994), Tang et al. (1996), McCarty et al. (1998) y Espitia et al. (2008). Lo anterior indica que los componentes del rendimiento de mayor importancia son las variables relacionadas con tamaño de capullo y número total de capullos por planta, y por ello son criterios de selección convenientes.

\section{CONCLUSIONES}

Las variedades convencionales Cian Precoz, Cian 95, Juárez 91, Laguna 89 y Nazas 87, a pesar de que no fueron superiores a Delta Pine, mostraron valores superiores en calidad de fibra para satisfacer las exigencias del

Cuadro 3. Correlación entre caracteres de rendimiento y calidad de fibra en seis variedades convencionales de algodón en la Comarca Lagunera, México, 2018.

\begin{tabular}{|c|c|c|c|c|c|c|c|c|c|c|c|c|}
\hline & REN & REF & REH & PCA & $\mathrm{CFI}$ & ISE & NCPP & APL & $\mathrm{PPH}$ & FIN & RES & LON \\
\hline REN & 1.00 & & & & & & & & & & & \\
\hline REF & $0.99 * *$ & 1.00 & & & & & & & & & & \\
\hline RES & $0.99 * \star$ & $0.99 * \star$ & 1.00 & & & & & & & & & \\
\hline PCA & $-0.46 * *$ & -0.46 & $-0.47 * \star$ & 1.00 & & & & & & & & \\
\hline CFI & 0.16 & 0.18 & 0.15 & -0.16 & 1.00 & & & & & & & \\
\hline ISE & $-0.34 * *$ & $-0.34 * *$ & $-0.33 * *$ & $0.41 * \star$ & $-0.47 \star \star$ & 1.00 & & & & & & \\
\hline NCPP & $0.91 * *$ & $0.90 * *$ & $0.91 * \star$ & $-0.47 \star \star$ & 0.15 & $-0.35 * \star$ & 1.00 & & & & & \\
\hline APL & $0.51 * *$ & $0.49 * *$ & $0.52 * *$ & $-0.22 * *$ & -0.1 & -0.14 & $0.59 * *$ & 1.00 & & & & \\
\hline $\mathrm{PPH}$ & $0.91 * *$ & $0.91 * *$ & $0.91 * *$ & $-0.43 * *$ & 0.17 & $-0.34 * \star$ & $0.98 * *$ & $0.55 * *$ & 1.00 & & & \\
\hline FIN & $0.55 * *$ & $0.55^{* *}$ & $0.55^{\star * \star}$ & $-0.39 * *$ & 0.31 * & -0.19 & $0.51 * *$ & $0.22 *$ & $0.52 * *$ & 1.00 & & \\
\hline RES & -0.15 & -0.15 & -0.16 & -0.04 & 0.13 & 0.19 & -0.1 & -0.01 & -0.1 & 0.17 & 1.00 & \\
\hline LON & $-0.21 *$ & $-0.21 *$ & $-0.21 *$ & $0.29 *$ & $-0.21 *$ & $0.27 \star \star$ & $-0.20 *$ & 0.03 & -0.2 & $-0.65 * \star$ & $-0.25 *$ & 1.00 \\
\hline
\end{tabular}

*: $\mathrm{P} \leq 0.05, * *: P \leq 0.01, \mathrm{REN}$ : rendimiento de algodón en hueso, REF: rendimiento de fibra, REH: rendimiento de semilla, PCA: peso de capullo, CFI: contenido de fibra, ISE: índice de semilla, NCPP. número de capullos por planta, APL: altura de planta, PPH: peso de fibra y semilla, FIN: finura, RES: resistencia de fibra, LON: longitud de fibra. 
mercado actual. Los materiales mencionados pueden ser una fuente importante de genes para calidad de fibra en programas de mejoramiento genético; sin embargo, es necesario definir zonas de adaptabilidad para mejorar los bajos rendimientos que se reportan en el presente trabajo.

\section{BIBLIOGRAFÍA}

Arias M. A., C. Mallén R., D. Garza R., J. B. Rentería A., L. Reyes M., P. Zamora M., M. R. Tovar G., S. Vargas M. y T. Gómez H. (2010) INIFAP; 25 años contribuyendo al desarrollo rural sustentable. Instituto Nacional de Investigaciones Forestales, Agrícolas y Pecuarias. México, D. F. 136 p.

Ashraf M. (2002) Salt tolerance of cotton: some new advances. Critical Reviews in Plant Sciences 21:1-30, https://doi. org/10.1080/0735-260291044160

CNSPA, Comité Nacional Sistema Producto Algodón (2012) Plan Rector Sistema Producto Algodón. Comité Nacional Sistema Producto Algodón, A. C. México, D. F. 52 p.

Dedaniya A. D. and K. V. Pethani (1994) Genetic variability, correlations and path analysis in deshi cotton (Gossypium arboreum L.). The Indian Journal of Genetics and Plant Breeding 54:229-234.

Escobedo-Salas M., U. Nava-Camberos, M. Ramírez-Delgado, J. H. EsparzaMartínez y M. Sepúlveda-Bojorquez (2004) Fenología, rendimiento, calidad de fibra y efectividad para el control de plagas de variedades transgénicas de algodonero, en la Comarca Lagunera. Revista Chapingo Serie Zonas Áridas 3:115-121.

Estrada T. 0. O., A. Palomo G., A. Espinoza B., S. A. Rodríguez H. y N. A. Ruiz T. (2008) Rendimiento y calidad de fibra del algodón cultivado en surcos ultra-estrechos. Revista Fitotecnia Mexicana 31(Núm. Esp. 3):79-83

Espinosa C. A., J. Piña R., A. C. de Oliveira y M. Mora V. (2004) Listado de variedades liberadas por el INIFAP de 1980 a 2003. Publicación Especial No. 2. Instituto Nacional de Investigaciones Forestales, Agrícolas y Pecuarias. México, D. F. 31 p.

Espitia C. M. M., H. Araméndiz T. y J. Cadena T. (2008) Correlaciones y análisis de sendero en algodón (Gossypium hirsutum L.) en el caribe colombiano. Revista Facultad Nacional de Agronomía Medellín 61:4325-4335.

FAO, Organización de las Naciones Unidas para la Agricultura y la Alimentación (2018) Cooperación sur-sur trilateral. Estudio nichos de mercados del algodón. Organización de las Naciones Unidas para la Alimentación y la Agricultura y Agencia Brasileña de Cooperación-Ministerio de Relaciones Exteriores. Santiago de Chile. $97 \mathrm{p}$.

Gaytán-Mascorro A., A. Palomo-Gil, D. G. Reta-Sánchez, S. Godoy-Ávila y E. A. García-Castañeda (2004) Respuesta del algodón cv. Cian Precoz 3 al espaciamiento entre surcos y densidad poblacional. I. Rendimiento, precocidad y calidad de fibra. Phyton 73:57-67.

Gould F., N. Blair, M. Reid, T. L. Rennie, J. Lopez and S. Micinski (2002) Bacillus thuringiensis-toxin resistance management: stable isotope assessment of alternate host use by Helicoverpa zea. Proceedings of National Academy of Sciences of the United States of America 99:16581-16586, https://doi.org/10.1073/ pnas. 242382499

Jost P. H. and J. T. Cothren (2000) Growth and yield comparisons of cotton planted in conventional and ultra-narrow row spacings. Crop Science 40:430-435, https://doi.org/10.2135/ cropsci2000.402430x

Longenecker D. E. and L. J. Erie (1968) Irrigation water management. In: Advances in Production and Utilization of Quality Cotton: Principles and Practices. F. C. Elliot, M. Hoover and W. K. Porter (eds.). Iowa State University Press. Ames, lowa, USA. pp:321345.

Martínez-Carrillo J. L. and N. Díaz-López (2005) Nine years of transgenic cotton in México, adoption and resistance management results. In: Proceedings of the Beltwide Cotton Conference. New Orleans, Louisiana. 4-7 January. National Cotton Council of America. Memphis, Tennessee, USA. pp:1368-1372.

Mastretta-Yanes A., M. R. Bellon, F. Acevedo, C. Burgeff, D. Piñero y J. Sarukhán (2019) Un programa para México de conservación y uso de la diversidad genética de las plantas domesticadas y sus parientes silvestres. Revista Fitotecnia Mexicana 42:321334, https://doi.org/10.35196/rfm.2019.4.321-334

McCarty J. C., J. N. Jenkins and J. Zhu (1998) Introgression of day-neutral genes in primitive cotton accessions: I. Genetic variances and correlations. Crop Science 38:1425-1428, https://doi. org/10.2135/cropsci1998.0011183X003800060002x

Mendelsohn M., J. Kough, Z. Vaituzis and K. Matthews (2003) Are Bt crops safe? Nature Biotechnology 21:1003-1009, https://doi. org/10.1038/nbt0903-1003

Nava-Camberos U., V. Ávila-Rodríguez, J. Maltos-Buendía, J. L. GarcíaHernández y J. L. Martínez-Carrillo (2018) Densidades y daños de insectos plaga en algodonero convencional y Bt en la Comarca Lagunera, México. Southwestern Entomologist 43:985-993. https://doi.org/10.3958/059.043.0415

Nuez F., M. J. Díez, J. V. Valcárcel, J. Cebolla-Cornejo, A. Pérez, S. Soler, ... and R. De Frutos (2008) Genetic resources of Lycopersicon at the Institute for the Conservation and Improvement of the Agrodiversity (COMAV). Acta Horticulturae 789:293-298, https://doi.org/10.17660/ActaHortic.2008.789.39

Palomo G. A., A. Gaytán M. y S. Godoy Á. (2003) Rendimiento, componentes del rendimiento y calidad de fibra del algodón en relación con la dosis de nitrógeno y la densidad poblacional. Revista Fitotecnia Mexicana 26:167-171

Palomo R. M., R. Rodríguez M. y M. Ramírez D. (2014) Picudo del algodonero y prácticas de manejo integrado. Folleto Técnico No. 29. Campo Experimental La Laguna, Instituto Nacional de Investigaciones Forestales, Agrícolas y Pecuarias. Matamoros, Coahuila. 42 p.

Palomo-Gil A., A. Gaytán-Mascorro, R. Faz-Contreras, D. G. Reta-Sánchez y E. Gutiérrez-del Río (2004) Rendimiento y calidad de fibra de algodón en respuesta al número de riegos y dosis de nitrógeno. Terra Latinoamericana 22:299-305.

Pérez M. C., M. R. Tovar G., Q. Obispo G., J. A. Ruíz C., L. Tavitas F., J. L. Jolalpa B. y F. J. Legorreta P. (2011) Los Recursos Genéticos del Algodón en México. Libro Técnico Núm. 5. Instituto Nacional de Investigaciones, Forestales, Agrícolas y Pecuarias. México, D. F. 120 p.

Pimentel G. F. (1985) Curso de Estadística Experimental. Universidad de São Paulo. São Paulo, Brasil. 466 p.

Poelham J. M. y D. Allen (2003) Mejoramiento Genético de las Cosechas. Segunda edición. Limusa. México, D. F. 512 p.

Rocha-Munive M. G., M. Soberón, S. Castañeda, E. Niaves, E. Scheinvar, L. E. Eguiarte, ... and V. Souza (2018) Evaluation of the impact of genetically modified cotton after 20 years of cultivation in Mexico. Frontiers in Bioengineering and Biotechnology 6:82, https://doi.org/10.3389/fbioe.2018.00082

SAGARPA, Secretaría de Agricultura, Ganadería, Desarrollo Rural, Pesca y Alimentación; FAO, Organización de las Naciones Unidas para la Alimentación y la Agricultura (2014) Análisis de la Cadena de Valor en la Producción de Algodón en México. SAGARPA-FAO. México, D. F. 98 p.

SAGARPA, Secretaría de Agricultura, Ganadería, Desarrollo Rural, Pesca y Alimentación (2016) Planeación Agrícola Nacional 2017-2030. Algodón Mexicano. Secretaría de Agricultura, Desarrollo Rural, Pesca y Alimentación. Ciudad de México. 11 p.

SAS Institute (2011) SAS/STAT® 9.3 9.3 User's Guide. SAS Institute. Cary, North Carolina, USA. 8621 p.

Scholl R. L. and P. A. Miller (1976) Genetic association between yield and fiber strength in upland cotton. Crop Science 16:780-783, https://doi.org/10.2135/cropsci1976.0011183X00160006001 Ox

SIAP, Servicio de Información Agroalimentaria y Pesquera (2016) Avance de siembras y cosechas. Resumen nacional por estado. Servicio de Información Agroalimentaria y Pesquera. Secretaría de Agricultura, Ganadería, Desarrollo Rural, Pesca y Alimentación. Ciudad de México. http://infosiap.siap.gob.mx:8080/agricola_ siap_gobmx/AvanceNacionalCultivo.do (Diciembre 2018)

SNICS, Servicio Nacional de Inspección y Certificación de Semillas (2012) Catálogo nacional de variedades vegetales. Servicio Nacional de Inspección y Certificación de Semillas. Secretaría de Agricultura, Ganadería, Desarrollo Rural, Pesca y Alimentación. México, D. F. 34 p.

Tang B., J. N. Jenkins, C. E. Watson, J. C. McCarty and R. G. Creech (1996) 
Evaluation of genetic variances, heritabilities, and correlations for yield and fiber traits among cotton $F_{2}$ hybrid populations. Euphytica 91:315-322, https://doi.org/10.1007/BF00033093

Traxler G., S. Godoy-Avila, J. Falck-Zepeda and J. J. Espinoza-Arellano (2003) Transgenic cotton in Mexico: A case study of the Comarca Lagunera. In: The Economic and Environmental Impacts of Agbiotech. A Global Perspective. N. Kalaitzandonakes (ed.). Springer. Boston, Massachusetts, USA. pp:183-202, https://doi. org/10.1007/978-1-4615-0177-0 10

USDA, United States Department of Agriculture (2017) Cotton Production Monthly Report. United States Department of Agriculture Washington, D. C. https://www.ams.usda.gov/market-news/ cotton (December 2018).

Vinent E. y 0. Fajardo (2007) Parámetros agrícolas de ocho cultivares de algodón (Gossypium hirsutum) obtenidos en Cuba. Ciencia e Investigación Agraria. 34:151-158, https://doi.org/10.4067/ S0718-16202007000200008

Worley S., H. H. Ramey, D. C. Harrell and T. W. Culp (1976) Ontogenetic model of cotton yield. Crop Science 16:30-34, https://doi. org/10.2135/cropsci1976.0011183X001600010008x

Wu K. M. and Y. Y. Guo (2005) The evolution of cotton pest management practices in China. Annual Review of Entomology 50:31-52, https://doi.org/10.1146/annurev.ento.50.071803.130349

Wu K. M., Y. H. Lu, H. Q. Feng, Y. Y. Jiang and J. Z. Zhao (2008) Suppression of cotton bollworm in multiple crops in China in areas with B toxin-containing cotton. Science 321:1676-1678, https://doi. org/10.1126/science. 1160550 
